\author{
ARTIGO \\ dol https://doi.org/10.22481/rpe.v16i43.6606
}

\title{
PROTOCOLO DE OBSERVAÇÃO DE AULA (POA) PARA O ENSINO ESPORTIVO: VALIDADE E CONFIABILIDADE
}

\author{
CLASS OBSERVATION PROTOCOL (COP) FOR SPORTS EDUCATION: VALIDITY \\ AND RELIABILITY
}

\author{
PROTOCOLO DE OBSERVACIÓN DE CLASE (POA) PARA LA EDUCACIÓN \\ DEPORTIVA: VALIDEZ Y CONFIABILIDAD
}

\author{
Maria Jullyanne Cavalcanti de Brito \\ Universidade Federal do Rio de Janeiro - Brasil \\ Antonio Jorge Gonçalves Soares \\ Universidade Federal do Rio de Janeiro - Brasil
}

Tony Meireles Santos

Universidade Federal de Pernambuco - Brasil

Vinícius de Oliveira Damasceno

Universidade Federal de Pernambuco - Brasil

\begin{abstract}
Resumo: O Programa Segundo Tempo criou o Protocolo de Observação de Aula (POA) a fim de analisar o quanto os professores conseguem materializar a metodologia do programa em suas aulas. $\mathrm{O}$ POA possui itens que contemplam aspectos que devem estar presentes em uma aula voltada para o ensino esportivo como um todo. Ele foi criado tanto para avaliar aulas, quanto para ser utilizado como um guia educativo para o planejamento e o melhoramento das mesmas. Considerando a necessidade de mensurar suas qualidades psicométricas, o presente estudo tem como objetivo testar a validade de critério, a consistência interna e a reprodutibilidade intra e interobservador das suas medidas. Para estabelecer a validade de critério, os escores das duas videoaulas foram comparados com o gabarito. Para a confiabilidade interobservador, foram comparados os escores que cada avaliador deu para as videoaulas. Quanto à confiabilidade intraobservador, foi usado o escore de uma segunda observação sobre a mesma videoaula em momentos diferentes. Após a análise dos resultados, as medidas do POA apresentaram boa confiabilidade (Alfa de Conbrach - 0,90; Reprodutibilidade Interobservador com CCI entre 0,89 e 0,91 e Reprodutibilidade Intraobservador com 11 dos 22 itens apresentando valor de $p$ significativo). Sobre a validade de critério, apenas cinco itens apresentaram concordância acima de 50\%. Desse modo, o POA traz boas perspectivas de aplicação e grande potencial de impacto no âmbito do ensino esportivo, contudo, parece importante que a capacitação para o seu uso seja reforçada, a fim de fortalecer os critérios de avaliação de aulas.
\end{abstract}

Palavras-chave: Avaliação Educacional; Psicometria; Reprodutibilidade dos testes. 
Abstract: The Programa Segundo Tempo, aiming to quantify the adhesion of its teachers to the metodology of the program in them classes, created the Classroom Observation Protocol (COP). The POA has items that contemplate aspects that should be present in a class focused on sports education. It was created both to evaluate classes and to be used as an educational guide to plan and improve classes. Due to the need to measure the psychometric qualities of this instrument, this study aims to test the validity criteria, internal consistency and intra and interobserver reproducibility of the measures obtained by the COP. To establish the validity criteria, two video classes were assessed, the scores obtained were compared with the template. Regarding the interobserver reliability, the values referring to the video classes observation performed by each evaluator were compared. Regarding to the intraobserver reliability, a calculation based in a second observation of the same evaluator about the same video class in a different moment was made. After the analysis of the results, the measures of the COP presented a good reliability (Cronbanch Alpha - 0,90; Interobserver reproducibility with ICC between 0,89 and 0,91 ; Intraobserver reproducibility with 11 of 22 items presenting significative $p$ value); nevertheless, concerning the validity criteria, only 5 items presented concordance above $50 \%$. Thus, the POA brings good prospects for application and great potential for impact in the field of sports education, but it is necessary a reinforcement its training, in order to strengthen the assessment criteria.

Keywords: Educational Measurement; Psychometrics; Reproducibility of Results.

Resumen: El Programa Segundo Tempo creó el Protocolo de Observación de Clase (POA) para analizar qué tan bien los maestros materializan la metodología del programa en sus clases. El POA tiene elementos que contemplan aspectos que deben estar presentes en una clase de educación deportiva. Por lo tanto, también fue creado para ser utilizado como una guía educativa para la planificación y mejora de las clases. Considerando la necesidad de medir sus cualidades psicométricas, el presente estudio tiene como objetivo probar la validez del criterio, la consistencia interna y la reproducibilidad intra e interobservador de sus medidas. Para establecer la validez del criterio, se compararon las puntuaciones de las clases de video con la plantilla. Para la confiabilidad entre observadores, se compararon los puntajes que cada evaluador dio para las clases de video. Para la confiabilidad intraobservador, se utilizó una segunda puntuación de observación en la misma clase de video en diferentes momentos. Después de analizar los resultados, las mediciones de POA mostraron buena confiabilidad (Alfa de Conbrach 0.90; Reproducibilidad interobservador con ICC entre 0.89 y 0.91 y Reproducibilidad intraobservador con 11 de los 22 ítems que muestran un valor p significativo). Con respecto a la validez del criterio, solo cinco ítems mostraron un acuerdo superior al 50\%. Por lo tanto, el POA ofrece buenas perspectivas de aplicación y un gran potencial de impacto en el campo de la educación deportiva, sin embargo, parece importante que se refuerce su capacitación, a fin de fortalecer los criterios para la evaluación.

Palabras clave: Evaluación Educacional; Psicometría; Reproducibilidad de los Resultados.

\section{Introdução}

Em alguns países, observações em sala de aula, pautadas em instrumentos protocolados para este fim, têm sido uma estratégia amplamente utilizada com o objetivo de obter informações sobre as oportunidades de aprendizagem às quais os alunos estão sendo submetidos por seus professores (CHEN; HENDRICKS; ARCHIBALD, 2011; FREDMAN, 2004; HAKEL; KOENIG; ELLIOTT, 2008; HILL et al., 2008; LORSON, 2010; MUIJS; REYNOLDS, 2003; PIANTA; HAMRE; MINTZ, 2012; SAWADA et al., 2002; STECHER et $a l .$, 2007). Em complemento, tais instrumentos também são úteis para fornecer feedback 
formativo para os professores, podendo promover melhorias na prática docente (BOSTON; CANDELA, 2018; COHEN et al., 2016).

Nesse sentido, o Protocolo de Observação de Aula (POA) foi criado com o intuito de estimar a qualidade de aulas esportivas e/ou pautadas no ensino da cultura corporal como um todo, podendo ser usado por pesquisadores, professores e demais profissionais da área de educação, desde que previamente treinados. As observações com base no POA podem ser realizadas a partir de vídeos, ou in loco, e possibilitam a análise de aspectos relacionados à interação entre professor e aluno, professor e materiais e/ou professor e conteúdo (SOARES et al., 2017).

O POA foi criado a partir de uma demanda do Programa Segundo Tempo (PST), um programa social, durante o contraturno escolar, que visa a democratização das práticas corporais por meio do atendimento de crianças e jovens em situações de vulnerabilidade social. Foi implementado em 2003, a partir de uma iniciativa do Ministério dos Esportes e, após 6 anos de existência, já havia atendido cerca de 1.300 municípios em todas as regiões do país, alcançando mais de 3 milhões de beneficiários (FILGUEIRA; PERIM; OLIVEIRA, 2009).

Considerando a grande abrangência do PST, bem como os recursos humanos e financeiros despendidos para sua implementação, é importante que, como qualquer programa social, este disponha de meios para verificar a qualidade do serviço ofertado. Dessa forma, o POA nasceu com o intuito de quantificar o nível de adesão das aulas dos professores do PST às diretrizes do programa, ou seja, verificar o quanto eles conseguem colocar em prática seus preceitos teóricos, tais como inclusão, maximização das experiências de aprendizagem, desafios, satisfação dos alunos com a aula etc. Com base nisso, as equipes colaboradoras do PST, pessoas responsáveis em realizar visitas às escolas e dar feedbacks aos professores, poderiam utilizar o POA em suas avaliações in loco para que, a partir das diagnoses obtidas, pudessem criar mecanismos a fim de manter e/ou aprimorar o que estava sendo materializado nas aulas (SOARES et al., 2017).

Em complemento, considerando que o POA possui itens que contemplam aspectos que essencialmente devem estar presentes em uma aula sob os moldes do PST, ou voltado para o ensino da cultura corporal como um todo, é possível que seja utilizado como um guia educativo para servir de referência à formação, ao planejamento e ao melhoramento de aulas pautadas no ensino esportivo, bem como da própria Educação Física (EF) escolar.

Uma aula de qualidade pensada sob os moldes do PST vai ao encontro das atuais perspectivas pedagógicas da $\mathrm{EF}$, tendo em vista que esta última também prega a não reprodução 
do esporte institucionalizado nas aulas, em que o objetivo central acaba se pautando no refinamento de técnicas ou fundamentos esportivos (ROTTMANN; RATTO, 2018). Portanto, a EF, assim como o PST, vem trabalhando para a democratização da prática esportiva, adotando estratégias que não classifiquem os alunos de acordo com seus méritos esportivos ou performáticos. Busca-se, portanto, incorporar práticas pedagógicas pautadas em estímulos afetivos, sociais, cognitivos e físicos, a fim de formar alunos que, além de estarem envolvidos com as atividades esportivas, sejam mais conscientes de seus papéis e responsabilidades na sociedade e na valorização do outro (DARIDO; OLIVEIRA, 2008; ROTTMANN; RATTO, 2018).

Sobre o ensino dos Jogos Esportivos Coletivos (JEC's), abordagens em que os fundamentos, gestos técnicos, táticas e ações físico-motoras são abordados em separado, dissociando drasticamente o saber fazer das razões para o fazer (SILVA, 1998), são interpretadas, a partir do POA, como inadequadas. Essa forma descontextualizada do ensino parte do pressuposto de que, ao aprender os conteúdos separadamente, a criança irá aplicar as supostas aprendizagens de forma conjunta durante o jogo propriamente dito (SCAGLIA et al., 2013; SILVA, 1998). Contudo, o jogo é um sistema complexo que se constitui do arranjo dialógico entre ordem, desordem e organização, cujas características não podem ser reduzidas a partes isoladas (LEONARDO; SCAGLIA; REVERDITO, 2009; REVERDITO; SCAGLIA, 2007). Dessa forma, ao ensinar um JEC, o professor não deve resumir-se ao ensino de gestos técnicos estereotipados. Além disso, os JEC's apresentam características semelhantes, no que diz respeito à sua referência estrutural (bola/implemento, alvo, zona de risco) e às suas referências funcionais (princípios operacionais e regras de ação), sendo necessário que os métodos adotados viabilizem o ensino do esporte a partir da lógica implícita no jogo (SCAGLIA et al., 2013).

Nesse sentido, a metodologia que o PST adota para o ensino do esporte é inspirada no modelo Teaching Grames for Undertanding (TGFU), que defende o ensino a partir de jogos modificados, ou seja, com graus de complexidade reduzidos, e que partam da consciência tática (KIRK; MACPHAIL, 2002; LIGHT; CURRY; MOONEY, 2014). Esse modelo prioriza a compreensão da lógica do jogo a fim de, posteriormente, desenvolver a técnica como ferramenta para processar as soluções motoras (GRAÇA; MESQUITA, 2007; GRECO; SILVA; SANTOS, 2009; MCBRIDE, 1991).

Ainda sobre a tecnologia educacional do PST, um dos aspectos imprescindíveis que devem estar presentes em suas aulas é a inclusão, 
entendida não apenas sob a perspectiva das deficiências físicas, mas também como outras diferenças individuais que podem proporcionar a exclusão (GOELLNER, 2009; MARQUES; CIDADE; LOPES, 2009). Dentre essas diferenças estão as de gênero, raça/etnia, geração, sexualidade, níveis de habilidades, entre outras. Sob tal perspectiva, é importante avaliar a capacidade do professor em incluir todos em todas as atividades, partindo do princípio de que incluir é proporcionar a mesma qualidade de execução da tarefa tanto para os mais, quanto para os menos habilidosos (GOELLNER, 2009; MARQUES; CIDADE; LOPES, 2009).

O POA procura contemplar todas essas nuances e, em estudo anterior, passou por fases que compõem o processo de construção de qualquer instrumento, que dizem respeito à elaboração dos itens, análise da validade de conteúdo e à pré-testagem (REICHENHEIM; MORAES, 2007; SOARES et al., 2017; STREINER, 2003). Nessa pré-testagem, os valores de concordância entre os membros da Equipe Pedagógica (EP) do PST sobre os itens do POA foram satisfatórios e, em seguida, o próximo passo necessário diz respeito à análise em maior escala das medidas que o instrumento oferece (REICHENHEIM; MORAES, 2007; STREINER, 2003).

A análise das medidas de um instrumento referem-se aos procedimentos que visam estabelecer a validade e a confiabilidade dos resultados obtidos através dele, a fim de verificar a taxa de erro presente na medida e a variação sistemática entre diferentes indivíduos confiabilidade; assim como o grau de confiança que poderemos atribuir às inferências que serão realizadas - validade (PASQUALI, 2003; STREINER; NORMAN, 2015). Nesse sentido, o objetivo do presente estudo foi testar a validade de critério (validade concorrente) e confiabilidade (consistência interna, reprodutibilidade intra e interobservador) das medidas obtidas pelo POA.

\section{Método}

Desenho do Estudo

O processo para estabelecer a validade concorrente entre os observadores e o gabarito, bem como a confiabilidade intra e interobservadores para as duas videoaulas, encontram-se descritos na Figura 1. 
Figura 1: Desenho do estudo

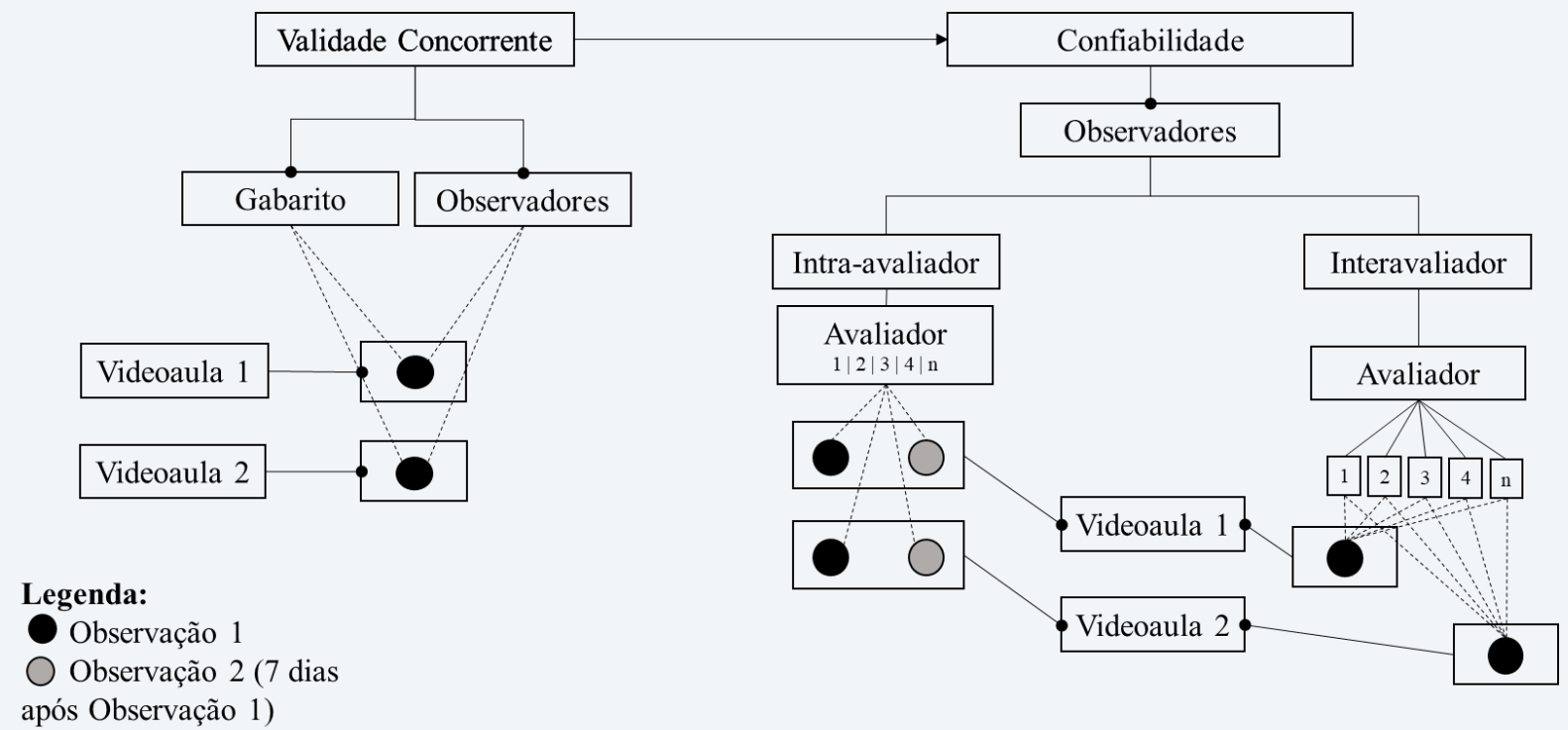

Fonte: Elaborada pelos autores.

Desse modo, para a validade concorrente foram comparadas as avaliações dos observadores com as do gabarito para as duas videoaulas. Para a confiabilidade intraobservador, foram comparadas as avaliações dos mesmos observadores para a mesma videoaula em momentos diferentes. Por fim, para a confiabilidade interobservador, foram analisados os dados de todos os observadores para as duas videoaulas ${ }^{1}$.

\section{$\underline{\text { Observadores }}$}

Os observadores foram recrutados por meio de uma divulgação nas redes sociais que se referia a uma capacitação online para professores de EF. Essa divulgação foi realizada nas redes sociais dos pesquisadores envolvidos e nas do PST. Inscreveram-se na capacitação graduandos, graduados e pós-graduados. Foram excluídos os participantes que não avaliaram nenhuma videoaula ou que apresentaram dados faltosos nas suas avaliações.

\section{$\underline{\text { Procedimentos }}$}

Capacitação: Para utilizar o POA, é indispensável que os observadores passem por uma capacitação. A estrutura da capacitação online do POA teve uma carga horária total de $5 \mathrm{~h}$ e está detalhada na Figura 2.

\footnotetext{
${ }^{1} \mathrm{O}$ estudo foi aprovado pelo Comitê de Ética do Centro de Ciências da Saúde da Universidade Federal de
} Pernambuco com parecer 040336.

Revista Práxis Educacional, Vitória da Conquista - Bahia - Brasil, v. 16, n. 43, p. 366-387, Edição Especial, 2020. 
Figura 2: Estrutura da capacitação do POA.

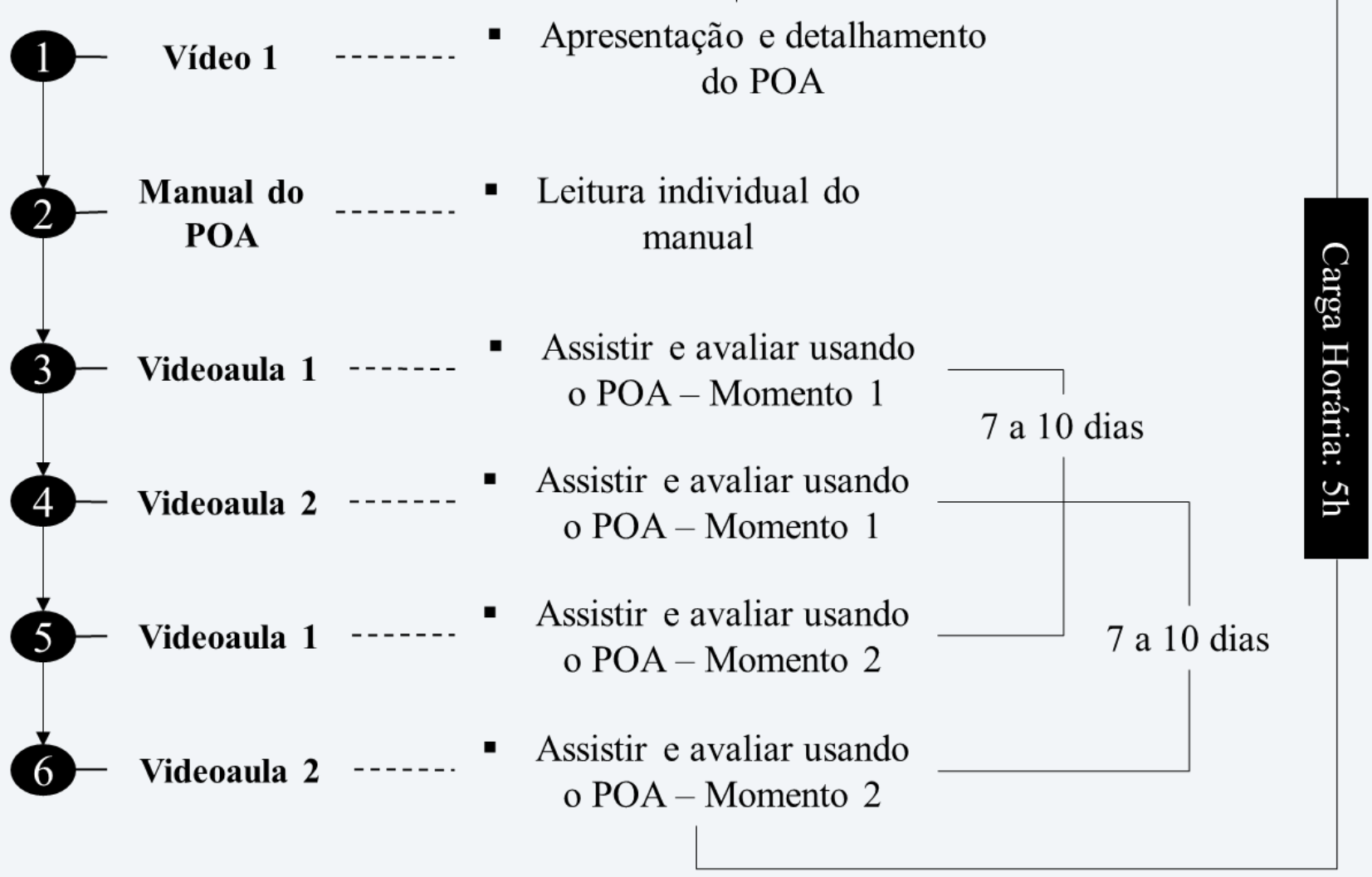

Fonte: Elaborada pelos autores.

Protocolo de Observação de Aula (POA): O POA possui 22 itens distribuídos entre cinco famílias: Indicadores de Planejamento, Indicadores de Conteúdo, Indicadores de Procedimentos Metodológicos, Indicadores de Avaliação e Adesão/inclusão dos alunos (Anexo 1). Esses itens, quando agrupados, geram o escore de Qualidade Geral da Aula (QGA), que reflete o grau de aderência da aula aos preceitos teóricos do PST. A QGA pode ser classificada como: adequada $(\geq 70 \%)$, parcialmente adequada $(\geq 50 \%$ e $<75 \%)$ ou inadequada $(<50 \%)$.

Videoaulas: As videoaulas que compõem a capacitação foram filmadas por uma única filmadora apoiada a um tripé. Ambos foram posicionados de modo que todo o espaço utilizado na aula pudesse ser visualizado. A voz do professor foi captada por um microfone do tipo lapela e os vídeos produzidos foram editados no Adobe Premier Pro CC 2015.

Videoaula 1: Foi filmada em um núcleo do PST vinculado ao Convênio Recife/Pernambuco. O espaço físico utilizado foi uma quadra ampla e coberta. O tema da aula foi o Handebol e o nível de adesão da aula aos pressupostos teóricos do PST, de 
acordo com o gabarito, foi de $61,4 \%$. Esse valor classifica uma aula como parcialmente adequada.

Videoaula 2: Também foi filmada em um núcleo do PST vinculado ao Convênio Recife/Pernambuco. O espaço físico foi um ambiente pequeno e não coberto situado na frente e na lateral da escola. O tema da aula foi o Voleibol e o nível de adesão da aula aos pressupostos teóricos do PST, de acordo com o gabarito, foi de 67,0\%. Esse valor classifica uma aula como parcialmente adequada.

Gabarito: O gabarito foi gerado a partir da avaliação individual das videoaulas por dois membros da Equipe de Avaliação Pedagógica do $\mathrm{PST}^{2}$, que, após reunião de consenso, acordaram sobre as classificações realizadas, gerando um valor único para cada item referente à aula avaliada.

Avaliação das videoaulas: Foram avaliadas individualmente pelos participantes durante a capacitação online. Cada videoaula foi avaliada duas vezes e em momentos diferentes, através de uma versão digital do POA que ficou disponível em um link gerado pelo Survey Monkey, criado especificamente para esse fim.

\section{$\underline{\text { Análise Estatística }}$}

As características dos sujeitos foram apresentadas pela estatística descritiva. Para a normalidade dos dados, foi adotado o teste Shapiro-Wilk. A confiabilidade intra e interobservador foi analisada adotando o Coeficiente de Correlação Intraclasse (CCI), modelo aleatório de duas vias, com medidas médias para concordância absoluta [CCI $(2, \mathrm{k})]$, a partir das medidas para cada item do POA e dos escores gerados. Os valores de CCI foram interpretados da seguinte maneira: valores $\leq 0,50$ (baixa reprodutibilidade), $>0,50$ até 0,75 (moderada reprodutibilidade), > 0,75 até 0,90 (alta reprodutibilidade) e valores $>0,90$ (excelente reprodutibilidade) (KOO; LI, 2016). Em complemento, para analisar a consistência interna, foi calculado o Alpha de Cronbach para cada família, bem como para todos os itens juntos. Para a validade concorrente, foi calculado o Índice de Concordância entre os valores obtidos em cada item pelos participantes e pelo gabarito.

\footnotetext{
${ }^{2}$ A Equipe de Avaliação Pedagógica é uma equipe vinculada hierarquicamente à Equipe Pedagógica do PST, que foi instituída para construir um modelo de avaliação pedagógica do programa.
} 


$$
\text { Índice de Concordância }=\frac{\text { Concordâncias }}{(\text { Concordâncias }+ \text { Discordâncias })} \times 100
$$

Além disso, para comparar os escores gerados pelos participantes e pelo gabarito, foi realizado o teste não paramétrico de Wilcoxon, tendo em vista que, no teste Shapiro-Wilk, os dados não apresentaram distribuição normal. Em complemento, foi feita a análise por inspeção visual do gráfico de Bland-Altman, ainda com os valores dos escores. Todas as análises foram realizadas no IBM SPSS, versão 23.0, e as figuras foram feitas pelo Graph Pad Prism, versão 5.01. O nível de significância adotado foi de $\mathrm{p}<0,05$.

\section{Resultados}

Participaram do estudo professores de vários estados do Brasil, cujo tempo de experiência, nível de formação acadêmica e outras informações encontram-se detalhados na Tabela 1.

Inscreveram-se na capacitação 122 pessoas. Destas, 119 iniciaram a capacitação (avaliação da videoaula 1) e apenas 50 finalizaram todas as etapas (avaliação da videoaula 1 e 2, em dois momentos diferentes), ou seja, o equivalente a $41 \%$ dos inscritos.

Os valores de Alfa de Cronbach para cada família foi: 1. Indicadores de Planejamento - 0,76; 2. Indicadores de Conteúdo - 0,86; 3. Indicadores de Procedimentos Metodológicos 0,84; 4. Indicadores de Avaliação - 0,82; e 5. Indicadores de Adesão/Inclusão dos alunos 0,73. Considerando todos os itens do POA juntos, o Alfa de Cronbach foi 0,94. Os valores obtidos na análise da reprodutibilidade interobservadores (Tabela 2) apresentaram valores de CCI classificados como alto (videoaula 1 - observação 1 e 2 e videoaula 2, observação 2) e como excelente (videoaula 2, observação 2). 
Tabela 1: Características do grupo.

\begin{tabular}{lcccc}
\hline & $\begin{array}{c}\text { Frequência } \\
\text { Absoluta }\end{array}$ & $\begin{array}{c}\text { Frequência } \\
\text { Absoluta } \\
\text { Acumulada }\end{array}$ & $\begin{array}{c}\text { Frequência } \\
\text { Relativa (\%) }\end{array}$ & $\begin{array}{c}\text { Frequência } \\
\text { Relativa } \\
\text { Acumulada (\%) }\end{array}$ \\
\hline Tempo de Experiência & 64 & & & \\
1 a 4 anos & 32 & 64 & 52,0 & 52,0 \\
5 a 10 anos & 11 & 107 & 26,1 & 78,1 \\
11 a 15 anos & 4 & 111 & 8,9 & 87,0 \\
16 a 20 anos & 5 & 116 & 3,4 & 90,4 \\
21 a 25 anos & 6 & 122 & 5,2 & 94,6 \\
+ 26 anos & & & & 100,0 \\
Nível de Formação Acadêmica & 64 & 64 & 52,5 & 52,2 \\
Graduação & 41 & 105 & 33,6 & 86,1 \\
Lato Sensu - Especialização & 8 & 113 & 6,6 & 92,7 \\
Stricto Sensu - Mestrado & 9 & 122 & 7,4 & 100,0 \\
Stricto Sensu - Doutorado & & & & \\
Participou de atividades do PST & 43 & 43 & 34,9 & 34,9 \\
Não & 79 & 122 & 65,1 & 100,0 \\
Sim & & & & \\
Videoaula Observada - & 119 & 119 & 43,3 & 43,3 \\
Momento Observado & 57 & 176 & 20,7 & 64,0 \\
Videoaula 1 - Momento 1 & 49 & 225 & 17,8 & 81,8 \\
Videoaula 2 - Momento 1 & 50 & 275 & 18,2 & 100,0 \\
Videoaula 1 - Momento 2 & & & \\
Videoaula 2 - Momento 2 & & & & \\
\hline
\end{tabular}

Fonte: Elaborada pelos autores.

Tabela 2: Reprodutibilidade interobservador para as videoaulas nos dois momentos de observação.

\begin{tabular}{lcllccr}
\hline \multirow{3}{*}{ Videoaulas } & \multicolumn{5}{c}{ Reprodutibilidade Interobservador } \\
\cline { 2 - 7 } & \multicolumn{4}{c}{ Observação 1 } & Observação 2 \\
\cline { 2 - 7 } & $\mathrm{n}$ & CCI & IC 95\% & $\mathrm{n}$ & CCI & IC 95\% \\
\hline Videoaula 1 & 119 & 0,90 & $0,86-0,92$ & 49 & 0,89 & $0,83-0,93$ \\
Videoaula 2 & 57 & 0,90 & $0,86-0,93$ & 50 & 0,91 & $0,85-0,95$ \\
\hline
\end{tabular}

Legenda. CCI: Coeficiente de Correlação Intraclasse. IC: Índice de Concordância.

Fonte: Elaborada pelos autores.

A Tabela 3 apresenta os valores de reprodutibilidade intraobservadores que foram calculados a partir dos valores que os observadores atribuíram para cada item. Nesta tabela, percebe-se que, dos 22 itens que compõem o instrumento, 11 apresentaram valores de $p$ significativos para ambas as videoaulas e a maioria desses itens demonstrou CCI classificado entre moderada e alta reprodutibilidade. Quanto aos demais itens, apenas três demonstraram valores insignificantes nas duas videoaulas (2. Encadeamento coerente das atividades e 14 . 
Ensino do jogo). Os oito itens restantes só apresentaram valores satisfatórios em uma das videoaulas.

Tabela 3: Reprodutibilidade intraobservador para videoaula 1 e videoaula 2 em momentos diferentes.

\begin{tabular}{|c|c|c|c|c|c|c|}
\hline \multirow[b]{3}{*}{ Famílias/Itens } & \multicolumn{3}{|c|}{ Videoaula 1} & \multicolumn{3}{|c|}{ Videoaula 2} \\
\hline & $\mathrm{O} 1$ & $\mathrm{O} 2$ & & $\mathrm{O} 1$ & $\mathrm{O} 2$ & \\
\hline & $\begin{array}{c}\text { Med. } \\
\text { (Interv. } \\
\text { Interq.) }\end{array}$ & $\begin{array}{c}\text { Med. } \\
\text { (Interv. } \\
\text { Interq.) }\end{array}$ & CCI;p & $\begin{array}{c}\text { Med. } \\
\text { (Interv. } \\
\text { Interq.) }\end{array}$ & $\begin{array}{c}\text { Med. } \\
\text { (Interv. } \\
\text { Interq.) }\end{array}$ & CCI;p \\
\hline \multicolumn{7}{|l|}{ Indicadores de Planejamento } \\
\hline 1. Objetivos da aula & $2(1-2)$ & $2(1-2)$ & 0,34 & $2(1-2)$ & $2(1-2)$ & $0,68 *$ \\
\hline 2. Encadeamento coerente das atividades & $2(1-2)$ & $2(1-2)$ & 0,33 & $2(1-2)$ & $2(1-2)$ & 0,52 \\
\hline 3. Ocupação do espaço físico disponível & $2(1-2)$ & $2(1-2)$ & $0,69 *$ & $2(1-2)$ & $2(1-2)$ & $0,65^{*}$ \\
\hline 4. Uso de materiais & $2(1-2)$ & $2(1-2)$ & 0,09 & $2(1-2)$ & $2(1-2)$ & 0,31 \\
\hline 5. Compatib. das atividades por faixa etária & $2(2-2)$ & $2(1-2)$ & $0,47^{*}$ & $2(1-2)$ & $2(1-2)$ & 0,36 \\
\hline \multicolumn{7}{|l|}{ Indicadores de Conteúdo } \\
\hline 6. Segurança no ensino dos conteúdos & $2(1-2)$ & $2(1-2)$ & 0,20 & $2(1-2)$ & $2(1-2)$ & $0,78^{*}$ \\
\hline 7. Linguagem adequada ao ensino & $2(1-2)$ & $2(1-2)$ & $0,65^{*}$ & $2(1-2)$ & $2(1-2)$ & 0,49 \\
\hline 8. Informações conceituais e procedimentais & $2(1-2)$ & $2(1-2)$ & $0,46^{*}$ & $2(1-2)$ & $2(1-2)$ & $0,56^{*}$ \\
\hline 9. Valores éticos e morais & $2(1-2)$ & $2(1-2)$ & $0,58 *$ & $2(1-2)$ & $2(1-2)$ & $0,77 *$ \\
\hline \multicolumn{7}{|c|}{ Indicadores de Procedimentos Metodológicos } \\
\hline 10. Relação com os alunos & $1(1-2)$ & $2(1-2)$ & $0,45^{*}$ & $2(1-2)$ & $2(1-2)$ & $0,58 *$ \\
\hline 11. Organização da turma & $2(1-2)$ & $2(1-2)$ & $0,54 *$ & $2(2-2)$ & $2(1-2)$ & 0,31 \\
\hline 12. Liderança na aula & $1(1-1)$ & $1(1-2)$ & $0,70^{*}$ & $2(1-2)$ & $2(1-2)$ & $0,69 *$ \\
\hline 13. Aquisição de habilidades técnicas & $2(1-2)$ & $1(1-2)$ & $0,69 *$ & $2(1-2)$ & $2(1-2)$ & 0,44 \\
\hline 14. Ensino do jogo & $2(1-2)$ & $2(1-2)$ & 0,09 & $2(1-2)$ & $1,5(1-2)$ & 0,22 \\
\hline 15. Tempo de experiência nas atividades & $1(1-2)$ & $1,5(1-2)$ & $0,57^{*}$ & $1(1-2)$ & $2(1-2)$ & $0,57^{*}$ \\
\hline 16. Comportamento estratégico e tático & $1(1-2)$ & $1(1-2)$ & 0,09 & $1(1-2)$ & $1(1-2)$ & $0,70^{*}$ \\
\hline 17. Replanejamento da(s) atividade(s) & $2(1-3)$ & $2(1-2)$ & $0,58 *$ & $2(1-2)$ & $2(1-2)$ & $0,74 *$ \\
\hline \multicolumn{7}{|l|}{ Indicadores de Avaliação } \\
\hline 18. Feedback individual & $1(0-2)$ & $1(1-2)$ & $0,63^{*}$ & $1(1-2)$ & $1(1-2)$ & $0,71 *$ \\
\hline 19. Feedback coletivo & $2(1-2)$ & $2(1-2)$ & $0,42^{*}$ & $2(1-2)$ & $1(1-2)$ & $0,71 *$ \\
\hline 20. Análise final da aula & $1,5(1-2)$ & $2(1-2)$ & $0,62^{*}$ & $2(1-2)$ & $2(1-2)$ & $0,84 *$ \\
\hline \multicolumn{7}{|l|}{ Indicadores Adesão/Inclusão dos alunos } \\
\hline 21. Satisfação com a aula & $2(1-2)$ & $2(1,75-2)$ & $0,54^{*}$ & $2(1-2)$ & $2(1-2)$ & $0,60 *$ \\
\hline 22. Inclusão & $2(1-2)$ & $2(1-2)$ & $0,50 *$ & $2(2-2)$ & $2(2-2)$ & 0,52 \\
\hline
\end{tabular}

Legenda. O1: Observação 1; O2: Observação 2. Med.: mediana; Interv, interq.: intervalo interquartil. CCI: Coeficiente de Correlação Intraclasse.

Fonte: Elaborada pelos autores. 
$\mathrm{Na}$ Tabela 4, podemos observar que os escores de todas as famílias apresentaram valores de CCI classificados entre moderados e altos. Desse modo, parece que as discordâncias presentes em alguns itens não influenciaram significativamente os escores gerais das famílias.

Tabela 4: Confiabilidade intraobservador para os escores das Famílias de Indicadores para as videoaulas 1 e 2 em ambos os momentos.

\begin{tabular}{|c|c|c|c|c|c|c|}
\hline \multirow{3}{*}{ Famílias da Indicadores } & \multicolumn{3}{|c|}{ Videoaula 1} & \multicolumn{3}{|c|}{ Videoaula 2} \\
\hline & \multirow{2}{*}{$\frac{\mathrm{O} 1}{\text { Média } \pm \mathrm{DP}}$} & \multirow{2}{*}{$\frac{\mathrm{O} 2}{\text { Média } \pm \mathrm{DP}}$} & \multirow{2}{*}{$\mathrm{CCI} ; \mathrm{p}$} & \multirow{2}{*}{$\frac{\mathrm{O} 1}{\text { Média } \pm \mathrm{DP}}$} & \multirow{2}{*}{$\frac{\mathrm{O} 2}{\text { Média } \pm \mathrm{DP}}$} & \multirow{2}{*}{-CCI; p } \\
\hline & & & & & & \\
\hline Indic. de Planejamento & $0,80 \pm 0,17$ & $0,81 \pm 0,16$ & $0,57^{*}$ & $0,83 \pm 0,16$ & $0,82 \pm 0,19$ & $0,68^{*}$ \\
\hline Indic. de Conteúdo & $0,78 \pm 0,22$ & $0,81 \pm 0,16$ & $0,63^{*}$ & $0,80 \pm 0,20$ & $0,82 \pm 0,22$ & $0,80^{*}$ \\
\hline Indic. de Procedimentos Metodológicos & $0,69 \pm 0,23$ & $0,73 \pm 0,21$ & $0,60^{*}$ & $0,74 \pm 0,21$ & $0,75 \pm 0,21$ & $0,80^{*}$ \\
\hline Indic. de Avaliação & $0,67 \pm 0,24$ & $0,74 \pm 0,21$ & $0,61^{*}$ & $0,73 \pm 0,23$ & $0,74 \pm 0,23$ & $0,81 *$ \\
\hline Indic. de Adesão/inclusão dos alunos & $0,82 \pm 0,22$ & $0,85 \pm 0,21$ & 0,54 & $0,87 \pm 0,19$ & $0,85 \pm 0,20$ & $0,68^{*}$ \\
\hline Qualidade Global da Aula & $0,75 \pm 0,18$ & $0,78 \pm 0,16$ & $0,61 *$ & $0,79 \pm 0,16$ & $0,80 \pm 0,18$ & $0,83^{*}$ \\
\hline
\end{tabular}

Legenda. Indic.: Indicadores; O1: Observação 1; O2: Observação 2. DP: Desvio-Padrão; CCI: Coeficiente de Correlação Intraclasse.

Fonte: Elaborada pelos autores.

Em contrapartida, quando comparamos as avaliações dos observadores com a do gabarito para uma das aulas, os resultados mostram-se divergentes, tendo em vista que, apenas em cinco itens, mais da metade dos observadores concordaram com o gabarito (Tabela 5). Além do Índice de Concordância, também foi realizado o teste de Wilcoxon para verificar o quanto os observadores responderam semelhante ao gabarito, porém, os valores obtidos não apresentaram nenhuma semelhança significativa entre um e outro. 
Tabela 5: Validade concorrente entre os observadores e o gabarito para a videoaula 1.

\begin{tabular}{lccc}
\multicolumn{1}{c}{ Famílias/Itens } & Gabarito & $\begin{array}{c}\text { Med. (Interv. } \\
\text { Interq.) }\end{array}$ & IC \\
\hline Indicadores de Planejamento & & & \\
1. Objetivos da aula & 1 & $2(0-2)$ & 0,38 \\
2. Encadeamento coerente das atividades & 1 & $2(0-2)$ & 0,41 \\
3. Ocupação do espaço físico disponível & 1 & $2(0-2)$ & 0,45 \\
4.Uso de materiais & 2 & $2(0-3)$ & 0,47 \\
5.Compatibilidade das atividades por faixa etária & 2 & $2(1-2)$ & $0,68^{*}$ \\
Indicadores de Conteúdo & & & \\
6. Segurança no ensino dos conteúdos & 1 & $2(0-2)$ & 0,36 \\
7. Linguagem adequada ao ensino & 1 & $2(0-2)$ & 0,33 \\
8. Informações conceituais e procedimentais dos conteúdos & 1 & $2(0-2)$ & 0,44 \\
9. Valores éticos e morais & 1 & $2(0-2)$ & 0,33 \\
Indicadores de Procedimentos Metodológicos & & & \\
10. Relação com os alunos & 1 & $1(0-2)$ & 0,44 \\
11. Organização da turma & 2 & $2(0-2)$ & $0,68^{*}$ \\
12. Liderança na aula & 2 & $1(0-2)$ & 0,43 \\
13. Aquisição de habilidades técnicas & 1 & $2(0-2)$ & 0,46 \\
14. Ensino do jogo & 1 & $2(0-3)$ & 0,37 \\
15. Tempo de experiência nas atividades & 1 & $1(0-2)$ & $0,51^{*}$ \\
16. Comportamento estratégico e tático & 1 & $1(0-3)$ & 0,45 \\
17. Replanejamento da(s) atividade(s) & 0 & $2(0-3)$ & 0,09 \\
Indicadores de Avaliação & & & \\
18. Feedback individual & 0 & $1(0-2)$ & 0,18 \\
19. Feedback coletivo & 1 & $2(0-2)$ & 0,38 \\
20. Análise final da aula & 1 & $1,5(0-2)$ & 0,45 \\
Indicadores de Adesão/inclusão dos Alunos & & & \\
21. Satisfação com a aula & 2 & $2(0-2)$ & $0,68^{*}$ \\
22. Inclusão & $2(0-2)$ & $0,63^{*}$ \\
\hline
\end{tabular}

Legenda: Med.: mediana; Interv, interq.: intervalo interquartil; IC: Índice de Concordância.

Fonte: Elaborada pelos autores.

Para avaliar a validade concorrente dos escores de cada família com o gabarito, também foi feita a análise por inspeção visual do gráfico de Bland-Altman, explicitada na Figura 3.

Nesta representação visual, podemos perceber que, nos Indicadores de Planejamento, Conteúdo e Adesão/Inclusão dos alunos, parte dos observadores tenderam a superestimar as ações do professor representado na videoaula, classificando-as mais positivamente do que o gabarito. O inverso ocorreu com os Indicadores de Procedimentos Metodológicos e Avaliação. Por fim, no escore de Qualidade Global da Aula, ainda percebe-se a tendência em superestimar as ações do professor. 
Figura 3: Análise Bland-Altman dos observadores versus o gabarito para os escores de cada família do POA.
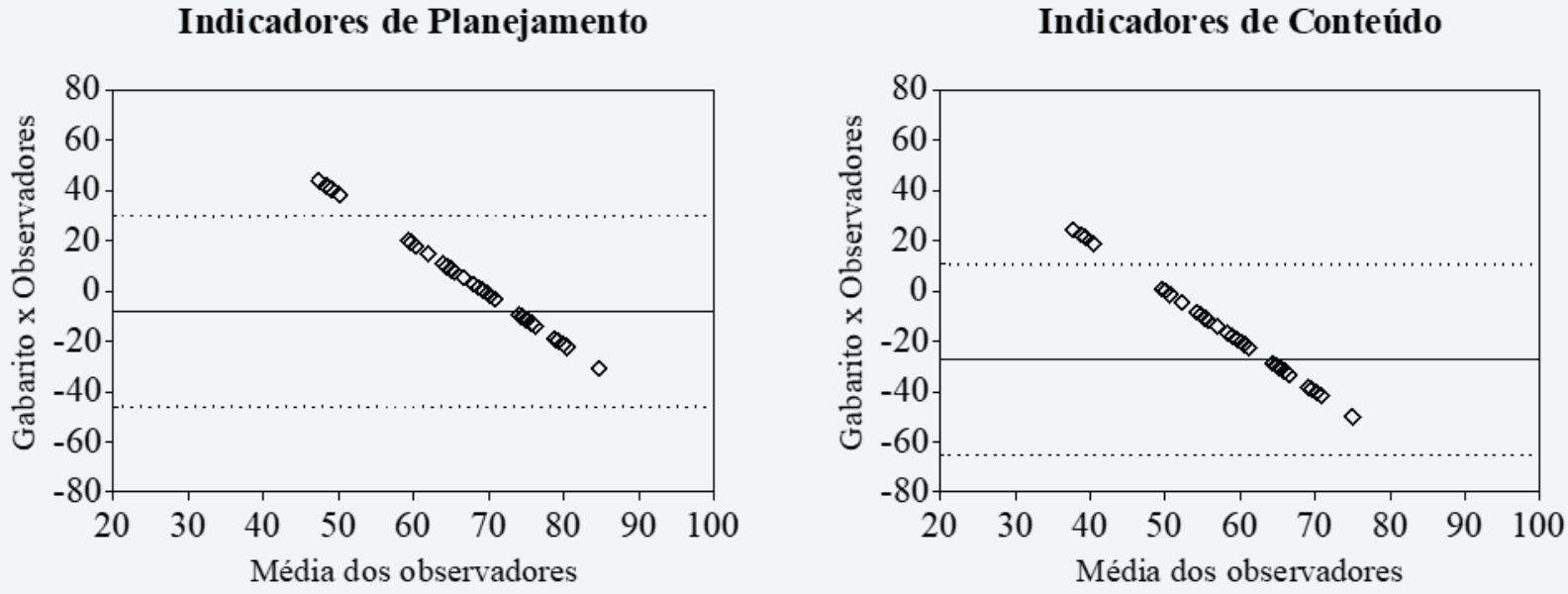

\section{Indicadores de Procedimentos Metodológicos}
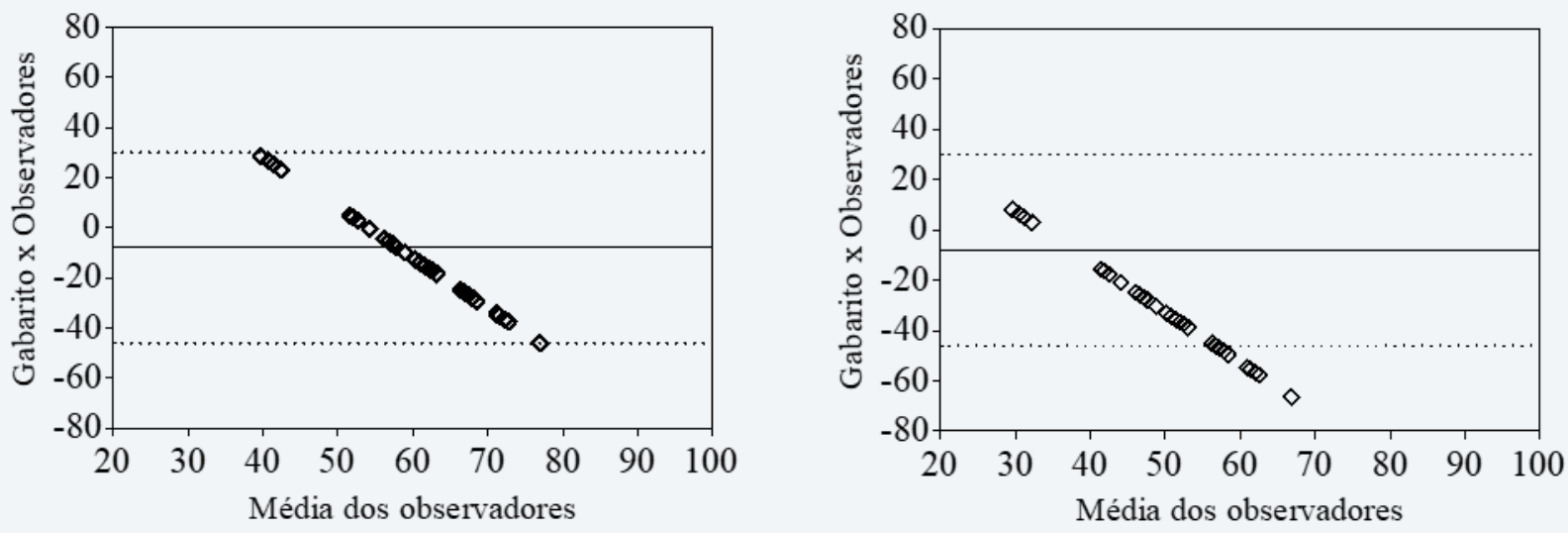

Indicadores de Adesão/Inclusão dos Alunos
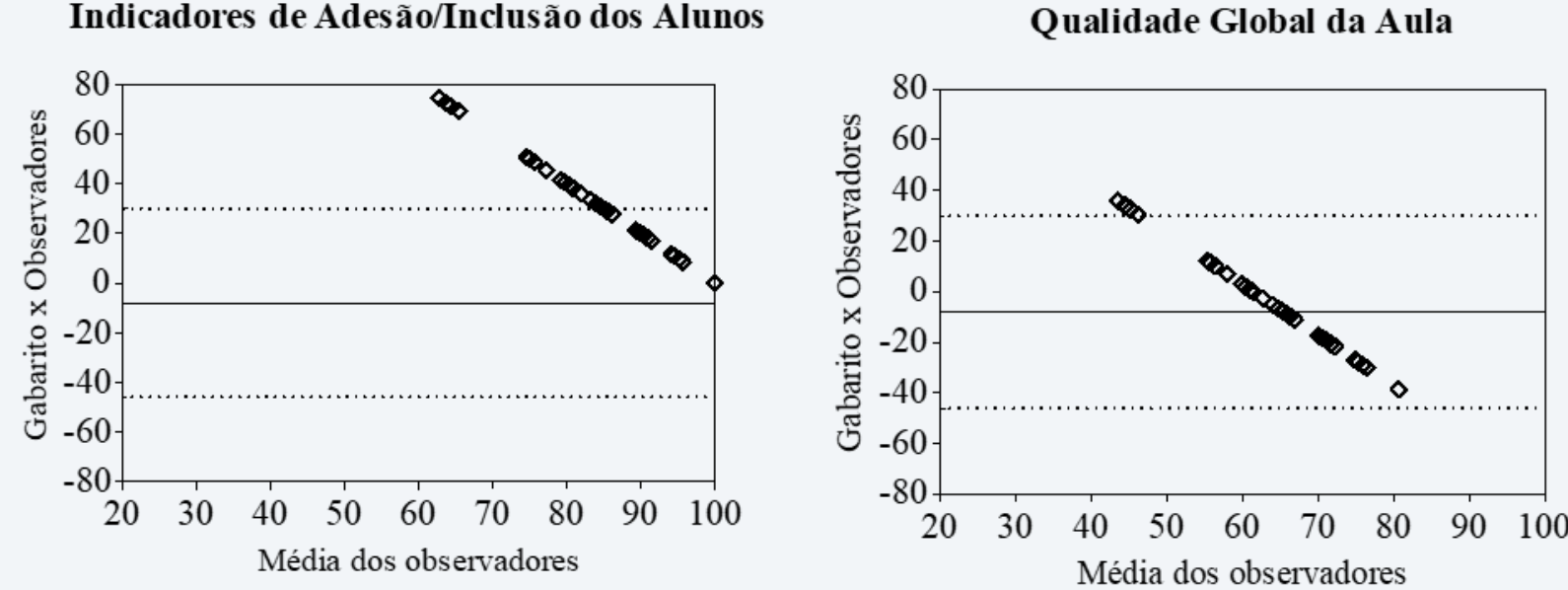

Fonte: Elaborada pelos autores. 


\section{Discussão}

O presente estudo se propôs a dar continuidade ao processo de validação das medidas do POA, compreendendo a validade de critério (observações dos participantes versus gabarito), consistência interna (grau de correlação entre os itens do instrumento), reprodutibilidade intraobservador (observação da mesma aula em momentos diferentes) e interobservador (observações de diferentes participantes sobre a mesma aula), a fim de garantir que o instrumento seja capaz de gerar resultados válidos durante futuras avaliações de aulas esportivas.

No que diz respeito à correlação entre os observadores para uma mesma videoaula, tanto neste estudo quanto no anterior, os valores de concordância foram significantes na maioria dos itens (SOARES et al., 2017). Dentre os estudos que utilizaram o Alfa de Cronbach para calcular a consistência interna de instrumentos de observação de aula de diversas disciplinas, o POA apresentou valores superiores ou semelhantes a esses, que tenderam a ficar entre 0,54 e 0,92. (CHEN; HENDRICKS; ARCHIBALD, 2011; HILL et al., 2008; PIANTA; HAMRE; MINTZ, 2012; RESNICK; MATSUMURA, 2006). Sendo assim, o POA demonstrou boa consistência interna.

Em relação às medidas intraobservador, os estudos que as realizaram o fizeram dentro de um intervalo de tempo bem maior, tendo como referência as estações do ano, além de utilizarem estatísticas diferentes, dificultando uma análise comparativa entre este e os demais estudos (HOFFMAN et al., 2004; PIANTA; HAMRE; MINTZ, 2012). Em complemento, a validade de critério em outros estudos foi tida como a tentativa de associar empiricamente os escores do novo instrumento com os já existentes, o que seria inviável com o POA, tendo em vista sua exclusividade. Ainda houve estudos que citaram um valor de critério para avaliação da aula, porém não foi explicitado como chegaram a este valor de critério nem descreveram a estatística utilizada para correlacionar com as respostas dos observadores (HOFFMAN et al., 2004; STECHER et al., 2007).

No presente estudo, percebeu-se que os resultados do CCI demonstraram que os observadores concordaram entre si tanto nas avaliações de ambas as videoaulas, como nas duas observações da mesma aula em momentos diferentes. Desse modo, o POA parece despertar interpretação semelhante entre diferentes observadores e em momentos distintos. Ainda assim, ao mesmo tempo em que os observadores concordaram entre si, eles se distanciaram dos valores de critério. Investigações já demonstraram que, mesmo após formações extensivas, diferenças importantes na forma como os observadores interpretam distintas situações ainda podem existir 
(KELCEY, 2013). Além disso, a experiência dos observadores é um fator que demonstra diferença significativa nos valores obtidos, tendo em vista que observadores experientes tendem a se aproximar mais das medidas de critério, aplicando as instruções dadas na capacitação com maior facilidade e confiança (ECKES, 2006).

Infelizmente, não nos atentamos a questionar os participantes sobre suas experiências prévias em observações protocoladas de aula, contudo, é provável que a maioria deles seja inexperiente, tendo em vista que essa não é uma habilidade comumente demandada no processo formativo de professores. Sendo assim, é provável que a possível inexperiência dos participantes tenha contribuído com parcela das discordâncias encontradas entre as observações e o gabarito.

Além disso, os observadores que participaram do estudo não foram o público-alvo inicial, ou seja, as Equipes Colaboradoras do PST, que são os responsáveis em realizar as avaliações in loco das aulas do programa. Sobre essas equipes, é provável que elas tenham um olhar mais acurado nas observações, tendo em vista sua intimidade com as diretrizes do PST, bem como sua experiência com outros modelos avaliativos que já foram adotados anteriormente. Neste estudo, optamos por testar o POA com um público mais variado, a fim de expandir o uso desses instrumentos para os demais campos educacionais que lidam com a educação esportiva, não o restringindo apenas ao uso do PST.

É importante considerar que o processo de validação não diz respeito apenas à integridade do instrumento, mas também a todo modelo de condução para sua aplicação, bem como ao perfil dos envolvidos nesse processo (STREINER; NORMAN, 2015). Desse modo, além dos dados obtidos pelo instrumento, estamos avaliando tanto os participantes do estudo, quanto o modelo de capacitação que foi adotado. Portanto, os resultados obtidos dizem respeito ao sucesso ou insucesso de todo esse processo, e não apenas do POA.

A capacitação foi montada em plataforma online e os observadores eram levados a assistir vídeos de aulas de EF. Essas videoaulas tinham de 40 a 45 minutos de duração e era solicitado que fossem assistidas novamente entre 7 e 10 dias depois. Esse é um procedimento que demanda tempo e disciplina e que, por ser realizado pelos observadores em suas próprias casas e no horário em que desejassem, não tínhamos como controlar a fidelidade do procedimento. Em complemento, tivemos uma perda amostral significativa, compreendendo $59 \%$ dos inscritos, o que é comum em pesquisas que são realizadas via plataformas online. Somado a isso, apesar da capacitação ser de apenas 5 horas, assistir ao mesmo vídeo mais de 
uma vez é um procedimento cansativo, o que deve ter sido desmotivador para os participantes e pode tê-los levado a não completar a capacitação.

Em relação ao formato da capacitação, seria interessante se tivéssemos proporcionado feedbacks sobre as avaliações realizadas, dando aos observadores momentos de tentativa e erro. Nesse caso, é importante que a capacitação seja reformulada, fornecendo momentos de avaliações prévias, seguidas de feedbacks quanto às divergências e proximidades encontradas na observação de determinada aula em relação ao gabarito, assim como ocorreu em um dos estudos com objetivos semelhantes a este (HOFFMAN et al., 2004).

De modo geral, o POA tem potencial para impactar de forma positiva as ações pedagógicas do PST, pois possibilitará a análise da qualidade das aulas do programa em larga escala a partir dos núcleos e das macrorregiões. A obtenção desses resultados auxiliará em intervenções mais direcionadas, a fim de viabilizar que as aulas sejam conduzidas de forma a proporcionar aos alunos vivências corporais mais próximas dos objetivos propostos pelo PST.

Entretanto, o POA transcende a medição de aulas, tendo em vista que também poderá ser utilizado como uma orientação para os professores, que poderão avaliar a sua própria aula e/ou planejá-las de acordo com os indicadores oferecidos pelo instrumento. Esse uso, segundo nossa hipótese, poderá ser válido não apenas para professores do PST, mas também por qualquer outro professor que lide com o ensino esportivo sob uma abordagem semelhante à do programa.

A EF, por exemplo, é uma disciplina obrigatória no currículo escolar, e que também se baseia no ensino dos esportes e das demais práticas da cultura corporal (BRASIL, 1997, 2000). No que diz respeito aos procedimentos metodológicos e à postura do professor frente aos alunos, percebe-se que as ações realizadas tanto pelo professor de EF quanto do PST possuem muitos aspectos equivalentes. Além disso, os conteúdos da EF estão voltados para a cultura corporal do movimento, englobando os esportes, jogos, lutas, ginásticas, atividades rítmicas expressivas e conhecimentos sobre o corpo (BRASIL, 1997, 2000), assemelhando-se aos conteúdos previstos para o PST, que também estão direcionados para as manifestações de cultura corporal (DARIDO; OLIVEIRA, 2008). Ademais, ambas as abordagens prezam pelos princípios de inclusão e de diversidade, além de referirem-se aos conteúdos embasados nas dimensões conceituais, procedimentais e atitudinais, partindo então de uma perspectiva muita mais ampla em relação aos saberes inerentes aos esportes e a outras manifestações culturais relacionadas ao corpo (BRASIL, 1997, 2000; DARIDO; OLIVEIRA, 2008). 
Desse modo, a condução de estudos futuros que verifiquem o impacto do POA na qualidade das aulas de professores de EF poderá proporcionar avanços no ensino esportivo em vários âmbitos (projetos sociais, aulas de EF etc.), beneficiando crianças, jovens e adultos de várias esferas econômicas. Além disso, o POA poderá estimular reflexões e inovações na forma como o ensino esportivo é teorizado e colocado em prática.

\section{Conclusão}

O POA demonstrou fácil aplicabilidade, tendo em vista os valores de confiabilidades obtidos. Porém, o modelo pelo qual o PST estruturou sua capacitação, bem como os participantes envolvidos, não favoreceu as análises de validade. Desse modo, é necessário que a capacitação online seja reformulada em alguns aspectos, tais como, oferecer momentos de tentativa e erro para os participantes, fornecendo feedbacks sobre as aulas avaliadas, a fim de que possam aplicar o critério de forma mais adequada. De modo geral, o POA traz boas perspectivas de aplicação e grande potencial de impacto no âmbito do ensino esportivo.

\section{REFERÊNCIAS}

BOSTON, Melissa; CANDELA, Amber. The Instructional Quality Assessment as a tool for reflecting on instructional practice. ZDM - Mathematics Education, Hamburgo, v. 50, n. 3, p. 427-444, jan./jun. 2018.

BRASIL. Parâmetros curriculares nacionais: Educação Física. Brasília: MEC/Secretaria de Educação Fundamental, 1997.

BRASIL. Parâmetros Curriculares Nacionais: Ensino Médio. Brasília: MEC/Secretaria de Educação Fundamental, 2000.

CHEN, Weiyun; HENDRICKS, Kristen; ARCHIBALD, Kelsi. Assessing pre-service teachers' quality teaching practices. Educational Research and Evaluation, Ann Arbor, v. 17, n. 1, p. 13-32, mai./jun. 2011. Disponível em: < https://www.researchgate.net/publication/232931305_Assessing_preservice teachers\%27_quality teaching_practices>. Acesso em: 28 mai. 2020.

COHEN, Julie; SCHULDT, Lorien Chambers; BROWN, Lindsay; GROSSMAN, Pam. Leveraging observation tools for instructional improvement: Exploring variability in uptake of ambitious instructional practices. Teachers College Record, Colúmbia, v. 118, n. 11, p. 136, 2016.

DARIDO, Suraya Cristina; OLIVEIRA, Amauri Aparecido Bássoli. Procedimentos Metodológicos para o Programa Segundo Tempo. In: OLIVEIRA, Amauri Aparecido Bássoli; 
PERIM, Gianna Lepre (Org.). Fundamentos Pedagógicos do Programa Segundo Tempo: da reflexão à prática. Maringá: Eduem, p. 207-236, 2008.

ECKES, Thomas. On commonn ground? How raters perceive scoring criteria in oral proficiency testing. In: BROWN, Anie; HILL, Kathryn (Org.). Tasks and Criteria in Performance Assessment. Cracóvia: Peter Lang, p. 43-73, 2006.

FILGUEIRA, Julio Cesar Monzú; PERIM, Gianna Lepre; OLIVEIRA, Amauri Aparecido Bássoli de. Apresentação. In: OLIVEIRA, Amauri Aparecido Bássoli; PERIM, Gianna Lepre (Org.). Fundamentos Pedagógicos do Programa Segundo Tempo: da reflexão à prática. Maringá: Eduem, p. 7-16, 2009.

FREDMAN, Tracy. Teacher Work Sample Methodology: Implementation and Practical Application in Teacher Preparation. Action in Teacher Education, Oklahoma, v. 26, n. 1, p. 3-11, jan. 2004.

GOELLNER, Silvana Vilodre. Corpo, gênero e sexualidade: educando para a diversidade. In: OLIVEIRA, Amauri Aparecido Bássoli; PERIM, Gianna Lepre (Org.). Fundamentos Pedagógicos do Programa Segundo Tempo: da reflexão à prática. Maringá: Eduem, p. 73$88,2009$.

GRAÇA, Amândio; MESQUITA, Isabel. A investigação sobre os modelos de ensino dos jogos desportivos. Revista Portuguesa de Ciências do Desporto, Porto, v. 7, n. 3, p. 401421, dez./set. 2007. Disponível em: <

http://www.scielo.mec.pt/scielo.php?script=sci_abstract\&pid=S164505232007000300014\&lng=pt\&nrm=i>. Acesso em: 28 mai. 2020.

GRECO, Pablo Juan; SILVA, Siomara; SANTOS, Lucídio Rocha. Organização e Desenvolvimento Pedagógico do Programa Segundo Tempo. In: OLIVEIRA, Amauri Aparecido Bássoli; PERIM, Gianna Lepre (Org.). Fundamentos Pedagógicos do Programa Segundo Tempo: da reflexão à prática. Maringá: Eduem, p. 163-206, 2009.

HAKEL, Milton; KOENIG, Judith Anderson; ELLIOTT, Stuart. Assessing accomplished teaching: advanced-level certification programs. Washington: The National Academies PRESS, 2008.

HILL, Heather; BLUNK, Merrie; CHARALAMBOS, Charalambous; LEWIS, Jennifer, PHELPS, Geoffrey; SLEEP, Laurie; BALL, Deborah Loewenberg. Mathematical knowledge for teaching and the mathematical quality of instruction: An exploratory study. Cognition and Instruction, Cambridge, v. 26, n. 4, p. 430-511, 2008. Disponível em: < https://www.researchgate.net/publication/247502158_Mathematical_Knowledge_for_Teachin g_and the_Mathematical_Quality_of_Instruction_An_Exploratory_Study>. Acesso em: 28 mai. 2020.

HOFFMAN, James; SAILORS, Misty; DUFFY, Gerald; BERETVAS, Natasha.The Effective Elementary Classroom Literacy Environment: examining the validity of the TEX-IN3 observation system. Journal of Literacy Research, Austin, v. 36, n. 3, p. 303-334, set. 2004. Disponível em: < https://journals.sagepub.com/doi/pdf/10.1207/s15548430jlr3603 33>. Acesso em: 28 mai. 2020. 
KELCEY, Ben. Noninvariant Measurement in Rater-Mediated Assessments of Teaching Quality. Society for Research on Educational Effectiveness. Disponível em: $<$ https://eric.ed.gov/?q=cross-classified+multilevel+model\&id=ED562804>. Acesso em: 27 mai. 2020.

KIRK, David; MACPHAIL, Ann. Teaching Games for Understanding and situated learning: Rethinking the Bunker-Thorpe model. Journal of Teaching in Physical Education, Loughborough, v. 21, n. 2, p. 177-192, jan. 2002. Disponível em: < https://www.researchgate.net/publication/44164225_Teaching_Games_for_Understanding_an d_Situated_Learning_Rethinking_the_Bunker-Thorpe_Model>. Acesso em: 28 mai. 2020.

KOO, Terry; LI, Mae. A Guideline of Selecting and Reporting Intraclass Correlation Coefficients for Reliability Research. Journal of Chiropractic Medicine, Nova York, v. 15, n. 2, p. 155-163, jul./nov. 2016.

LEONARDO, Lucas; SCAGLIA, Alcides José; REVERDITO, Riller Silva. O ensino dos esportes coletivos: metodologia pautada na família dos jogos. Motriz Revista de Educação Física. (Impr.), São Paulo, v. 15, n. 2, p. 236-246, abr./jun. 2009. Disponível em: < https://www.google.com/url?sa=t\&rct=j\&q=\&esrc $=$ s\&source=web\&cd=\&cad=rja\&uact=8\&v ed=2ahUKEwigqry xNbpAhVeCrkGHa7ZAhgQFjAAegQIBBAB\&url=http\%3A\%2F\%2Fw ww.periodicos.rc.biblioteca.unesp.br\%2Findex.php\%2Fmotriz\%2Farticle\%2Fview\%2F2177 \%2F2285\&usg=AOvVaw1x-GoLKb4B96EZ6WoOQIgJ>. Acesso em: 28 mai. 2020.

LIGHT, Richard; CURRY, Christina; MOONEY, Amanda. Game sense as a model for delivering quality teaching in physical education. Asia-Pacific Journal of Health, Sport and Physical Education, Vitória, v. 5, n. 1, p. 67-81, fev. 2014.

LORSON, Kevin. Making Teacher Work Samples "Work" for Accreditation. Journal of Physical Education, Recreation \& Dance, v. 81, n. 2, p. 54-58, fev. 2010. Disponível em: $<$ https://www.tandfonline.com/doi/abs/10.1080/07303084.2010.10598436>. Acesso em: 28 mai. 2020.

MARQUES, Alexandre Carriconde; CIDADE, Ruth Eugênia; LOPES, Kathya Augusta Thomé. Questões da deficiência e as ações no Programa Segundo Tempo. In: OLIVEIRA, Amauri Aparecido Bássoli; PERIM, Gianna Lepre (Org.). Fundamentos Pedagógicos do Programa Segundo Tempo: da reflexão à prática. Maringá: Eduem, p. 115-162, 2009.

MCBRIDE, Ron. Critical Thinking: an overview with implications for Physical Education. Journal of Teaching in Physical Education, Texas, v. 11, n. 2, p. 112-125, 1991.

MUIJS, Daniel; REYNOLDS, David. Student Background and Teacher Effects on Achievement and Attainment in Mathematics: A Longitudinal Study. Educational Research and Evaluation, Coventry, v. 9, n. 3, p. 289-314, ago. 2003.

PASQUALI, Luiz. Introdução à Análise Fatorial. In: Psicometria: teoria dos testes na psicologia e na educação. 2 ed. Petrópolis: Vozes, 2003.

PIANTA, Robert C.; HAMRE, Bridget; MINTZ, Susan. Classroom Assessment Scoring System [CLASS] manual, Upper Elementary. v. 6, p. 115-126, 2012. Disponível em: <http://cdn2.hubspot.net/hubfs/336169/Technical_Manual.pdf>. Acesso em: mai. 2020. 
REICHENHEIM, Michael Eduardo; MORAES, Claudia Leite. Operacionalização de adaptação transcultural de instrumentos de aferição usados em epidemiologia. Revista de Saúde Pública, Rio de Janeiro, v. 41, n. 4, p. 665-673, jan./abr. 2007. Disponível em: < https://www.scielo.br/scielo.php?pid=S003489102007000400024\&script=sci_arttext\&tlng=pt>. Acesso em: 28 mai. 2020.

RESNICK, Lauren; MATSUMURA, Lindsay Clare. Mathematics Instruction in Urban Middle Schools: a pilot study of the instructional quality assessment. Los Angeles: The Regents of the University of California, 2006.

REVERDITO, Riller Silva; SCAGLIA, Alcides José. A gestão do processo organizacional do jogo: uma proposta metodológica para o ensino dos jogos coletivos. Motriz, Rio Claro, v. 13, n. 1, p. 51-63, jan./mar. 2007. Disponível em: < http://multimidia.curitiba.pr.gov.br/2016/00178172.pdf>. Acesso em: 28 mai. 2020.

ROTTMANN, Hans Gert; RATTO, Cleber Gibbon. Educação Física: repensando práticas pedagógicas, sociabilidades e esporte na cultura contemporânea. Práxis Educacional, Vitória da Conquista. v. 14, n. 28, p. 374-393, abr./jun. 2018. Disponível em: < http://periodicos2.uesb.br/index.php/praxis/article/view/3476>. Acesso em: 28 mai. 2020.

SAWADA, Dayo; TURLEY, Jeff; FALCONER, Katheleen; BENFORD, Russell; BLOOM, Irene. Measuring Reform Practices in Science and Mathematics Classrooms: the reformed teaching observation protocol. School Science and Mathematics, Arizona, v. 102, n. 6, p. 245-253, out. 2002.

SCAGLIA, Alcides José; REVERDITO, Riller Silva; LEONARDO, Lucas; LIZANA, Cristian Javier Ramirez. O ensino dos jogos esportivos coletivos: as competências essenciais e a lógica do jogo em meio ao processo organizacional sistêmico. Movimento, Campinas, v. 19, n. 4, p. 227-249, out./dez. 2013. Disponível em: <

https://seer.ufrgs.br/Movimento/article/view/37893 >. Acesso em: 28 mai. 2020.

SILVA, Júlio Manuel Garganta da. O ensino dos jogos desportivos coletivos: perspectivas e tendências. Movimento, Campinas, v. 4, n. 8, p. 19-27, jan. 1998. Disponível em: < https://seer.ufrgs.br/Movimento/article/view/2373 >. Acesso em: 28 mai. 2020.

SOARES, Antonio Jorge Gonçalves; BRITO, Maria Jullyanne Cavalcanti de; SANTOS, Tony Meireles. OLIVEIRA, Amauri Aparecido Bassoli; CARDOSO, Marcelo Silva; VIEIRA, José Luiz Lopes; COSTA, Luciane Cristina Arantes da; DAMASCENO, Vinícius de Oliveira. Concepção e Validação do Protocolo de Observação de Aula (POA) do Programa Segundo Tempo. Journal of Physical Education, Maringá, v. 28, n. 1, p. 1-12, dez. 2017. Disponível em: < http://www.periodicos.uem.br/ojs/index.php/RevEducFis/article/view/39949>. Acesso em: 28 mai. 2020.

STECHER, Brian; BORKO, Hilda; KUFFNER, Karin; MARTINEZ, Felipe; ARNOLD, Suzanne; BARNES, Dionne; CREIGHTON, Laura; GILBERT, Lou. Using Artifacts to Describe Instruction: lessons learned from studying reform-oriented instruction in middle school mathematics and science. Los Angeles: The Regents of the University of California, 2007. 
STREINER, David. Being Inconsistent About Consistency: when coefficient alpha does and doesn't matter. Journal of Personality Assessmente, Filadélfia, v. 80, n. 3, p. 217-222, jun. 2003.

STREINER, David L.; NORMAN, G. R. Health measurement scales a practical guide to their development and use. 5. ed. USA: OUP Oxford, 2015.

\section{SOBRE OS AUTORES:}

\section{Maria Jullyanne Cavalcanti de Brito}

Doutoranda em Educação pela Universidade Federal do Rio de Janeiro (UFRJ/BR). Membra do Laboratório de Pesquisa em Educação do Corpo (LABEC). Professora de Educação Física do ensino fundamental no município de Jaboatão dos Guararapes/PE. E-mail: Jullyanne_cavalcanti@hotmail.com

iD https://orcid.org/0000-0002-5692-614X

\section{Antonio Jorge Gonçalves Soares}

Doutor em Educação Física pela Universidade Gama Filho (UGF/BR), com pós-doutorado pela Faculdade de Desporto da Universidade do Porto (FADEUP/PT). Professor do Programa de Pós-Graduação em Educação da Universidade Federal do Rio de Janeiro (UFRJ). Líder do Laboratório de Pesquisa em Educação do Corpo (LABEC). Pesquisador do CNPq. Bolsista de Produtividade. E-mail: ajgsoares@gmail.com

(iD https://orcid.org/0000-0001-7769-9268

\section{Tony Meireles Santos}

Doutor em Educação Física pela Universidade Gama Filho (UGF/BR). Professor do Programa de Pós-Graduação em Educação Física da Universidade Federal de Pernambuco (UFPE/BR). É coordenador do Núcleo de Investigação em Performance e Saúde e membro do grupo de pesquisa Performance e de Estudos em Psicofisiologia do Exercício. E-mail: tonymsantos@gmail.com.

(iD) https://orcid.org/0000-0002-5242-0117

\section{Vinícius de Oliveira Damasceno}

Doutor em Ciências da Saúde pela Universidade Federal de Minas Gerais (UFMG/BR). Professor do Programa de Pós-Graduação em Educação Física da Universidade Federal de Pernambuco (UFPE/BR). E-mail: vinicius.damasceno@gmail.com

(iD) https://orcid.org/0000-0003-0577-9204

Recebido em: 28 de maio de 2020 